\title{
SMARCA5 interacts with NUP98-NSD1 oncofusion protein and sustains hematopoietic cells transformation
}

\author{
Zivojin Jevtic ${ }^{1}$, Vittoria Matafora ${ }^{1}$, Francesca Casagrande ${ }^{2}$, Fabio Santoro ${ }^{3}$, Saverio Minucci ${ }^{3,4}$, \\ Massimilliano Garre' ${ }^{2}$, Milad Rasouli ${ }^{5}$, Olaf Heidenreich ${ }^{5}$, Giovanna Musco ${ }^{6}$, Jürg Schwaller ${ }^{7 *}$ and \\ Angela Bachi ${ }^{1 *}$ (D)
}

\begin{abstract}
Background: Acute myeloid leukemia (AML) is characterized by accumulation of aberrantly differentiated hematopoietic myeloid progenitor cells. The karyotyping-silent NUP98-NSD1 fusion is a molecular hallmark of pediatric AML and is associated with the activating FLT3-ITD mutation in $>70 \%$ of the cases. NUP98-NSD1 fusion protein promotes myeloid progenitor self-renewal in mice via unknown molecular mechanism requiring both the NUP98 and the NSD1 moieties.

Methods: We used affinity purification coupled to label-free mass spectrometry (AP-MS) to examine the effect of NUP98-NSD1 structural domain deletions on nuclear interactome binding. We determined their functional relevance in NUP98-NSD1 immortalized primary murine hematopoietic stem and progenitor cells (HSPC) by inducible knockdown, pharmacological targeting, methylcellulose assay, RT-qPCR analysis and/or proximity ligation assays (PLA). Fluorescence recovery after photobleaching and b-isoxazole assay were performed to examine the phase transition capacity of NUP98-NSD1 in vitro and in vivo.

Results: We show that NUP98-NSD1 core interactome binding is largely dependent on the NUP98 phenylalanineglycine (FG) repeat domains which mediate formation of liquid-like phase-separated NUP98-NSD1 nuclear condensates. We identified condensate constituents including imitation switch (ISWI) family member SMARCA5 and BPTF (bromodomain PHD finger transcription factor), both members of the nucleosome remodeling factor complex (NURF). We validated the interaction with SMARCA5 in NUP98-NSD ${ }^{+}$patient cells and demonstrated its functional role in NUP98-NSD1/FLT3-ITD immortalized primary murine hematopoietic cells by genetic and pharmacological targeting. Notably, SMARCA5 inhibition did not affect NUP98-NSD1 condensates suggesting that functional activity rather than condensate formation per se is crucial to maintain the transformed phenotype.

Conclusions: NUP98-NSD1 interacts and colocalizes on the genome with SMARCA5 which is an essential mediator of the NUP98-NSD1 transformation in hematopoietic cells. Formation of NUP98-NSD1 phase-separated nuclear condensates is not sufficient for the maintenance of transformed phenotype, which suggests that selective targeting of condensate constituents might represent a new therapeutic strategy for NUP98-NSD1 driven AML.
\end{abstract}

\footnotetext{
*Correspondence: j.schwaller@unibas.ch; angela.bachi@ifom.eu

${ }^{1}$ Functional Proteomics group, IFOM-FIRC Institute of Molecular Oncology, Milan, Italy

${ }^{7}$ Department for Biomedicine, University Children Hospital, Basel, Switzerland

Full list of author information is available at the end of the article
} original author(s) and the source, provide a link to the Creative Commons licence, and indicate if changes were made. The images or other third party material in this article are included in the article's Creative Commons licence, unless indicated otherwise in a credit line to the material. If material is not included in the article's Creative Commons licence and your intended use is not permitted by statutory regulation or exceeds the permitted use, you will need to obtain permission directly from the copyright holder. To view a copy of this licence, visit http://creativecommons.org/licenses/by/4.0/. The Creative Commons Public Domain Dedication waiver (http://creativeco mmons.org/publicdomain/zero/1.0/) applies to the data made available in this article, unless otherwise stated in a credit line to the data. 
Keywords: NUP98-NSD1, SMARCA5, Acute myeloid leukemia, Phase-separation, Interactomics

\section{Background}

Acute myeloid leukemia (AML) is characterized by accumulation of aberrantly differentiated hematopoietic myeloid progenitor cells [1]. Chromosomal translocations involving the Nucleoporin 98 gene (NUP98) result in $>30$ distinct gene fusions found in different hematological malignancies including AML $[2,3]$. Functional studies have shown that NUP98 fusion proteins promote myeloid progenitor self-renewal and prevent their differentiation through mechanisms that are heavily influenced by the fusion partner [2,3]. Moreover, several recent studies suggested that transformation of myeloid progenitors directly depends on phase transition capacity of NUP98 fusion proteins, mediated by the disordered $\mathrm{N}$-terminal NUP98 moiety which is shared among all fusions [4-6].

The karyotyping-silent NUP98-NSD1 fusion is a molecular hallmark of pediatric AML present with high white blood cell counts, and is generally associated with additional mutations of which the activating FLT3-ITD mutation is found in $>70 \%$ of the cases [7, 8]. The NUP98-NSD1 fusion carries the N-terminus of NUP98, including its disordered phenylalanine-glycine (FG) repeats and GLEBS domains, and the C-terminus of NSD1 bearing five plant homeodomains (PHD) and one PHD finger-like Cys-His-rich domain $(\mathrm{C} 5 \mathrm{HCH}$ or PHD6), one proline-tryptophan-proline domain (PWWP), and the lysine methyltransferase SET domain [9]. Retroviral NUP98-NSD1 promotes myeloid progenitor self-renewal in mice by maintaining the expression of HoxA7, HoxA9, HoxA10, HoxB4, HoxB5, and Meis1 genes via a mechanism requiring both the NUP98 and the NSD1 moieties $[10,11]$. It has been shown that the NUP98 moiety interacts with transcriptional coactivators EP300 and KMT2A (also known as MLL1) [10, 11]. PHD5 and $\mathrm{C} 5 \mathrm{HCH}$ domains from the NSD1 moiety mediate binding of genomic loci, while their transcriptionally active state is ensured by SET domain-catalyzed H3K36me2 [10]. Despite these insights, the molecular mechanism of NUP98-NSD1 driven leukemogenesis remains poorly characterized.

Herein, we determined the NUP98-NSD1 nuclear interactome, and examined its dependency on specific domains of the fusion protein. We found that core interactome binding was largely dependent on the FG repeat domains which mediate formation of liquid-like phaseseparated NUP98-NSD1 nuclear condensates. We identified condensate constituents including imitation switch (ISWI) family member SMARCA5 and BPTF (bromodomain PHD finger transcription factor), both members of the nucleosome remodeling factor complex (NURF), and validated the interaction with SMARCA5 remodeler in NUP98-NSD1 ${ }^{+}$patient cells. Furthermore, we demonstrated an important role of SMARCA5 in self-renewal of NUP98-NSD1 immortalized murine hematopoietic cells and regulation of HoxA9 and Meis1 proto-oncogene expression. As SMARCA5 knockdown/pharmacologic targeting did not affect formation of the NUP98-NSD1 nuclear condensates, we propose that a fully functional interactome is necessary to maintain the transformed state.

\section{Materials \& methods}

\section{Cell lines and lysate preparations}

Cell lines were acquired from the American Type Culture Collection (ATCC) and cell culture media were obtained from Lonza. All media were supplemented with 10\% fetal bovine serum (FBS). HEK-293 cells were grown in DMEM, while 32Dcl3 cells were grown in RPMI containing $10 \mathrm{ng} / \mathrm{ml}$ of murine IL-3. The cells were grown at $37^{\circ} \mathrm{C}$ with $5 \%$ of $\mathrm{CO}_{2}$ and maintained according to manufacturer instructions. For transfection experiments, HEK293 cells were plated at $70 \%$ confluency. We utilized the calcium-phosphate method [12] with $10 \mu \mathrm{g}$ of corresponding plasmids. The medium was changed after $8 \mathrm{~h}$ and the cells were incubated for $36 \mathrm{~h}$. Nuclear extracts were made using Dignam's protocol [13]. For stable transfection of 32Dcl3 cells the NEPA21 Electroporator was used (NEPA GENE). 32Dcl3 cells and immortalized NUP98NSD1/FLT3-ITD primary murine cells were lysed using $4 \mathrm{X}$ Laemmli buffer, after which the lysate was boiled at $95^{\circ} \mathrm{C}$ for $5 \mathrm{~min}$. Patient cells were obtained from the lab of Prof. Olaf Heidenreich at Princes Maxima Center for Pediatric Oncology in Utrecht, The Netherlands. NUP98$\mathrm{NSD}^{+}$patient cells were grown in StemSpan ${ }^{\mathrm{TM}}$ (STEMCELL Technologies) supplemented with $10 \mathrm{ng} / \mathrm{mL}$ IL-3, $10 \mathrm{ng} / \mathrm{mL}$ FLT3 ligand, $10 \mathrm{ng} / \mathrm{mL}$ GM-CSF, $150 \mathrm{ng} / \mathrm{mL}$ SCF, $100 \mathrm{ng} / \mathrm{mL}$ TPO.

\section{Immunoprecipitation}

Nuclear extracts were resuspended in IP buffer $(10 \mathrm{mM}$ Tris HCL pH 7.6, $150 \mathrm{mM} \mathrm{NaCl}$, 0.4\% NP-40, 1X EDTAfree Roche protease inhibitors) to a final concentration of $1 \mathrm{mg} / \mathrm{ml}$. Protein concentration was determined using Bradford assay. FLAG-tagged proteins were immunoprecipitated using anti-FLAG M2-affinity gel (SigmaAldrich). For co-immunoprecipitation experiments that were followed by Western blotting, $40 \mu \mathrm{l}$ of M2 affinity gel was added to $5 \mathrm{mg}$ of nuclear extracts previously 
resuspended in IP buffer. If the experiment was followed by mass-spectrometry analysis, the same amount of M2 affinity gel was added to $10 \mathrm{mg}$ of nuclear extracts/ IP buffer. The reaction was incubated for $10 \mathrm{~min}$ at $4^{\circ} \mathrm{C}$, after which the beads were washed two times with "washing buffer-1" (10 mM Tris $\mathrm{HCl}$ pH7.6, $500 \mathrm{mM}$ $\mathrm{NaCl}, 0.5 \% \mathrm{NP}-40,1 \mathrm{X}$ EDTA-free Roche protease inhibitors), two times with "washing buffer-2" (PBS, 0.5\% NP-40, 1X EDTA-free Roche protease inhibitors) and once with PBS. The elution was done using 3XFLAG peptide (\#F4799, Sigma-Aldrich) which was resuspended in elution buffer $(50 \mathrm{mM}$ Tris $\mathrm{HCl} \mathrm{pH} \mathrm{7.6,300} \mathrm{mM} \mathrm{NaCl,}$ $1 \%$ glycerol,1X EDTA-free Roche protease inhibitors) to a final concentration of $50 \mathrm{ng} / \mathrm{ml}$. Eluted proteins were reduced using $4 \mathrm{X}$ Laemmli buffer and boiled at $95^{\circ} \mathrm{C}$.

\section{Sample preparation and mass spectrometry analysis}

Immunoprecipitated samples were run on a $4-12 \%$ BisTris Gel (Thermo Scientific) and subjected to Coomassie staining. Protein bands were excised from the SDS-gel. Excised bands were chopped into pieces of approximately $1 \mathrm{x} 1 \mathrm{mM}$. Gel particles were transferred into a $1.5 \mathrm{ml}$ Eppendorf tube where the reduction of proteins' disulfide bridges was performed using $10 \mathrm{mM}$ DTT in $100 \mathrm{mM}$ $\mathrm{NH}_{4} \mathrm{HCO}_{3}$ at $56^{\circ} \mathrm{C}$ for $55 \mathrm{~min}$, while protein alkylation was performed using $55 \mathrm{mM}$ iodoacetamide in $100 \mathrm{mM}$ $\mathrm{NH}_{4} \mathrm{HCO}_{3}$ for $20 \mathrm{~min}$ at the room temperature. Proteins were digested with trypsin $(0.1 \mu \mathrm{g} / \mu \mathrm{l}$ in $100 \mathrm{mM}$ $\mathrm{NH}_{4} \mathrm{HCO}_{3}$ ) and incubated at $37^{\circ} \mathrm{C}$ overnight. After digestion, peptides were extracted from the gel pieces using acetonitrile and $5 \%$ formic acid. Peptide extracts were then purified using the StageTip procedure [14], dried in a SpeedVac and resuspended in 1\% trifluoroacetic acid (TFA) before mass spectrometry analysis. Five microliter of purified peptides were injected into the chromatographic system (Thermo Scientific, EASY-nLC 1200 Liquid Chromatography system), and separated on the self-made capillary column (ReproSil-Pur 120 C18-AQ, $1.9 \mu \mathrm{m}$, Dr. Maisch GmbH, $360 \times 0.075 \mathrm{mM}$ ). Peptide separation was achieved on a linear gradient from $95 \%$ solvent A ( $2 \%$ acetonitrile, $0.1 \%$ formic acid) to $55 \%$ solvent B ( $80 \%$ acetonitrile, $0.1 \%$ formic acid) over $75 \mathrm{~min}$ and from 55 to $100 \%$ solvent B in $3 \mathrm{~min}$ at a constant flow rate of $0.25 \mu \mathrm{l} / \mathrm{min}$ on UHPLC Easy-nLC 1000 (Thermo Scientific) where the LC system was connected to a $23-\mathrm{cm}$ fused-silica emitter of $75 \mu \mathrm{m}$ inner diameter (New Objective, Inc. Woburn, MA, USA), packed in-house with ReproSil-Pur C18-AQ $1.9 \mu \mathrm{m}$ beads (Dr Maisch Gmbh, Ammerbuch, Germany) using a highpressure bomb loader (Proxeon, Odense, Denmark).

The LC system was coupled to the Thermo Scientific ${ }^{\mathrm{TM}}$ Q Exactive ${ }^{\mathrm{TM}} \mathrm{HF}$ hybrid quadrupole-Orbitrap mass spectrometer. The total run time including sample loading and column reconditioning was $60 \mathrm{~min}$. The Q Exactive HF was operated in a DDA top 20 method with an MS survey scan resolution setting of 60,000 and MS/MS resolution setting of 17,500 . Peptide fragmentation was performed with an NCE (normalized collision energy) of 25 and an isolation window of $2.0 \mathrm{~m} / z$. Automatic gain control target value and maximum ion injection times were $3 \times 10^{5}$ and $60 \mathrm{~ms}$ for MS, and $10^{5}$ and $60 \mathrm{~ms}$ for MS/MS. Dynamic exclusion was enabled with an exclusion duration of 20s. The mass spectrometry proteomics data have been deposited to the ProteomeXchange Consortium via the PRIDE partner repository with the dataset identifier PXD026020 [15].

\section{Proteomics analysis}

All raw files were processed using MaxQuant [16] (Version 1.5.2.8) against a UniProtKB/Swiss-Prot human database containing 85.336 entries (downloaded on 02.02.2017). Carbamidomethylation was set as fixed modification while methionine oxidation and N-terminal acetylation were searched as variable modifications. Statistical analysis was done in Perseus [17] using two-sample t-tests with Benjamini-Hochberg correction set at $\mathrm{FDR}=0.05$. Volcano plots were created to display the results of t-testing, with FDR value of 0.05 , and S0 value set at 5 . For the visualization of this comparison, volcano plots and heatmap were produced using the inhouse written $\mathrm{R}$ script on which $\mathrm{x}$-axis shows the ratio (fold change) between $\log 2$ transformed LFQ values of the proteins bound by NUP98-NSD1 and mutated forms, while on the $\mathrm{y}$-axis - $\log 10$ transformed $p$ values (obtained in $\mathrm{t}$-test) were plotted.

\section{ChIP-Seq read alignment}

FLAG-NUP98-NSD1 genome binding sites were obtained from a previously published ChiP-seq dataset (GSE112928), expressed in primary murine hematopoietic cells. Reads were aligned to the reference genome (mm10) using bowtie2 algorithm, and peak calling was performed after duplicate filtering using MACS2. To identify NUP98-NSD1 target genes, peaks were intersected with an interval of $+/-1.5 \mathrm{~kb}$ around the genomic transcription start sites, which yielded 553 gene targets of the fusion protein. Similarly, SMARCA5 peaks were obtained from the public ChIP-seq dataset acquired in human leukemic K562 cell line (replicates GSM2424122 and GSM2424123), mapped using hg38 human reference genome and intersected with an interval of $+/-1.5 \mathrm{~kb}$ around the TSS of the human genome. Acquired SMARCA5 target genes (10,377 genes) were converted into corresponding murine orthologs using an ad-hoc script in R. Gene Set Enrichment Analysis was performed on shared target genes using enrichr package 
in $\mathrm{R}$ assessing the enrichment for the (GO) Biological Processes.

\section{Immunoblot analysis}

Western blotting was performed using standard protocols. Primary antibodies were diluted in 5\% milk solution, according to manufacturer's instructions. We used antiFLAG (\#F7425, Sigma-Aldrich), anti-CHD4 (\#ab72418, Abcam), anti-BPTF \# A300-973A, Fortis Life Sciences), anti-SMARCA5 (\#A301-017A-T, Fortis Life Sciences), anti-KDM1 (\#ab37165, Abcam), anti-HDAC2 (\#ab12169, Abcam). Primary antibodies were incubated for $2 \mathrm{~h}$ at RT with the nitrocellulose membranes. Washing of the nitrocellulose membranes was done 3 times for $5 \mathrm{~min}$, after which secondary antibodies (anti-mouse or antirabbit, Bio-Rad) were incubated for $1 \mathrm{~h}$. After three more washes, membranes were developed using the ECL-Plus Western Blotting Reagent (Amersham Biosciences).

\section{Immunofluorescence}

Transfected HEK293 cells were seeded on glass coverslips that were previously placed in 24 well plates. 32D cl3, NUP98-NSD1/FLT3-ITD, and patient NUP98$\mathrm{NSD}^{+}$cells were attached onto fibronectin-coated glass coverslips $(13 \mathrm{~mm}$ in diameter). Cells were fixed with $4 \%$ paraformaldehyde (Sigma-Aldrich) and permeabilized with $0.2 \%$ TritonX-100 (in PBS). Blocking was done using 10\% horse serum. Cells were incubated for $1 \mathrm{~h}$ at room temperature with primary antibodies, diluted at optimized concentrations in 5\% horse serum. Following primary antibodies' incubation (Anti-FLAG M2 mouse (\#F1804, Sigma), 1:2000), the appropriate secondary antibodies were used and incubated with cells for $45 \mathrm{~min}$ (Alexa Fluor- coupled antibodies from Jackson ImmunoResearch. Finally, cells were counterstained with DAPI to visualize the nuclei and then mounted on microscope slides using glycerol. The samples were investigated by confocal microscopy performed on a Leica TCS SP5 confocal laser scanning microscope (Leica Microsystem, Heidelberg, Germany). The images were acquired with an HCX PL APO 63X/1.4NA oil immersion objective. The software used for all acquisitions was LAS AF (Leica). Raw images were then analyzed using Fiji software $(\mathrm{NIH}$, Bethesda, USA). The figures were assembled using Adobe Illustrator.

\section{Proximity ligation assay (PLA)}

The Duolink In Situ Far Red Starter Kit Mouse/Rabbit (Sigma-Aldrich) was used for the detection of examined interactions. Cells were attached onto the fibronectincoated coverslips during overnight incubation at $37^{\circ} \mathrm{C}$ with $5 \%$ of $\mathrm{CO}_{2}$. The following day, the cells were gently washed in PBS and fixed using 4\% paraformaldehyde
(Sigma-Aldrich). Permeabilization was performed using $0.2 \%$ TritonX-100 in PBS at room temperature for $10 \mathrm{~min}$. Then, the cells were gently washed in PBS at room temperature twice. Cells were placed in an in-house made dark and humid chamber. Duolink Blocking solution was used for the blocking step, which was performed at $37^{\circ} \mathrm{C}$ for $60 \mathrm{~min}$. Anti-FLAG, SMARCA5 and BPTF antibodies were diluted in Duolink Antibody diluent solution (both in same Eppendorf tube) at the concentration 1:2000, NSD1 (C-term) antibody (\#N312/10, NeuroMab) was similarly diluted in Duolink Antibody diluent at the concentration 1:1000, and NUP98 (N-term) antibody (\#M1-26400, antibodies-online.com) at the concentration 1:500. After the blocking, incubation with primary antibodies was performed for $60 \mathrm{~min}$ at room temperature. Subsequent steps were performed following manufacturer's instructions. Finally, imaging of these samples was performed on Leica TCS SP5 confocal laser scanning microscope with an HCX PL APO 63X/1.4NA oil immersion objective, and Nikon Crest V3 Spinning Disc Confocal microscope. Images were acquired with LAS AF software (SP5) and NFI software (Nikon) analyzed using Fiji software.

\section{Fluorescent recovery after photobleaching (FRAP analysis)} FRAP experiments were performed on a Leica TCS SP8 confocal microscope, using HC PL APO CS2 63X/1,40 objective, managed by Leica LasX software. 10 Pre-bleach images were acquired at maximum speed using the white light laser set at $488 \mathrm{~nm}$ and $2 \%$ power. Then, after bleaching the fluorescence signal at background level using the same laser at maximum power, post-bleach images were acquired according to the following scheme: 30 images at maximum speed, 30 images every $1 \mathrm{~s}, 50$ images every $2 \mathrm{~s}$. Only complete recovery curves were used for the analysis. All of the steps in the analysis were performed using the Leica LasX software, including background subtraction, correction for imaging photobleaching, normalization, curve fitting (single exponential) and mathematical data collection (recovery half-time).

\section{Biotinylated isoxazole-mediated precipitation}

Biotinylated isoxazole (b-isox, Sigma-Aldrich), was resuspended in DMSO. Briefly, HEK293 cells were lysed

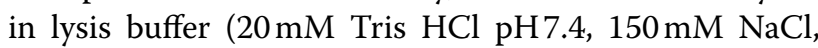
$5 \mathrm{mM} \mathrm{MgCl} 2,0.5 \% \mathrm{NP}-40$ and $10 \%$ glycerol, supplemented with 1X EDTA-free Roche protease inhibitors for $1 \mathrm{~h}$ at $4{ }^{\circ} \mathrm{C}$ ) [18]. After $30 \mathrm{~min}$ centrifugation at maximum speed at $4{ }^{\circ} \mathrm{C}$, protein supernatant was collected, and b-isox was added to $30 \mu \mathrm{M}$ final concentrations. The reaction was incubated for $1 \mathrm{~h}$ at $4{ }^{\circ} \mathrm{C}$. The lysates were then centrifuged for $15 \mathrm{~min}$ at $14000 \mathrm{rpm}$ at $4{ }^{\circ} \mathrm{C}$. Pellets were washed twice in lysis buffer and then reduced in $4 \mathrm{X}$ 
Laemmli buffer, while the supernatants were immediately reduced and boiled at $95^{\circ} \mathrm{C}$. Supernatants and pellets were analysed by $4-12 \%$ Bis-Tris Gel (Thermo-Scientific) and Western blotting was performed using standard protocols.

\section{Retroviral transduction of murine hematopoietic stem and progenitor cells}

All mouse experiments were in adherence to Swiss animal welfare laws and approved by the Swiss Cantonal Veterinary Office of Basel Stadt (licence no.2087).

For the infection of primary murine HSCs, 6-8 weeks old C57BL/6 mice were primed with $150 \mathrm{mg} / \mathrm{kg}$ 5-Fluorouracil (5-FU, Sigma, St Louis, MO) for 6 days. Bone marrow from femurs and tibias was extracted and flushed with the medium using sterile techniques. Bone marrow cells were incubated overnight in RPMI medium containing $10 \mathrm{ng} / \mathrm{ml}$ recombinant murine Interleukin-3 (IL-3, Peprotech), $50 \mathrm{ng} / \mathrm{ml}$ recombinant murine stem cell factor (SCF, Peprotech), and $10 \mathrm{ng} / \mathrm{ml}$ recombinant murine Interleukin-6 (IL-6, Peprotech), $100 \mathrm{U} / \mathrm{ml}$ penicillin/streptomycin (Gibco-BRL, Gaithersburg, MD), 20\% FBS, $2 \mathrm{mM}$ glutamine, in RPMI 1640 medium (Lonza). Retroviral constructs expressing NUP98-NSD1 (pMSCV$n e o-N U P 98-N S D 1)$ and FLT3-ITD-GFP (pMSCVFLT3-ITD-IRES-EGFP) were transiently transfected in Phoenix-ECO cells using calcium phosphate transfection method. Viral supernatants were collected upon $48 \mathrm{~h}$ of transfection, filtered using $0.45 \mu \mathrm{m}$ filters and then concentrated using Vivaspin 20 (Sartorius) columns at $3000 \mathrm{rpm}$, for $2 \mathrm{~h}$ at $4{ }^{\circ} \mathrm{C}$. Concentrated viral supernatant volumes of $200 \mu \mathrm{l}$ were snap-frozen in liquid nitrogen and kept at $-80^{\circ} \mathrm{C}$. The transduction of $5 \times 10^{5}$ primary murine HSC cells was performed in $2 \mathrm{ml}$ volume (in 12 well plate) comprised of concentrated viral supernatants mixed 1:1 (500 $\mu \mathrm{l}$ each) and $1 \mathrm{ml}$ of RPMI 1640 supplemented with $50 \mathrm{ng} / \mathrm{ml} \mathrm{SCF}, 10 \mathrm{ng} / \mathrm{ml} \mathrm{IL-3,} \mathrm{and} 10 \mathrm{ng} / \mathrm{ml}$ IL- 6 and polybrene at $8 \mu \mathrm{g} / \mathrm{ml}$. The plates were centrifuged for $90 \mathrm{~min}$ at $2500 \mathrm{rpm}$ at $30^{\circ} \mathrm{C}$. Upon centrifugation, the cells were incubated for $4 \mathrm{~h}$, after which $1 \mathrm{ml}$ of RPMI 1640 medium supplemented with IL-3, IL-6, and SCF was added. Transduced cells were expanded in the RPMI1640 medium supplemented with IL-3 $(10 \mathrm{ng} / \mathrm{ml})$, IL-6 $(5 \mathrm{ng} / \mathrm{ml})$, and SCF $(20 \mathrm{ng} / \mathrm{ml})$, and G418 concentration of $0.8 \mu \mathrm{g} / \mathrm{ml}$.

\section{Flow cytometry and single-cell sorting}

For the GFP sorting and single-cell GFP sorting experiments, cell pellets were washed in PBS, then resuspended in sterile $1 \%$ BSA (in PBS) and subjected to FACSAria IIU (Beckton Dickinson) instrument, previously adjusted for corresponding analyses. Doublet events were excluded, while GFP positive singlets were separated from other cells. For the analysis of cell-surface marker expression, cell pellets were washed using $1 \%$ BSA (in PBS), blocking was performed in 5\% BSA (in PBS) for $45 \mathrm{~min}$ at the room temperature. Upon blocking, cell pellets were resuspended in $100 \mu$ of primary antibody solutions, and incubated for $30 \mathrm{~min}$ on ice. Then, collected cell pellets were washed two times in $1 \mathrm{ml}$ PBS. Fixation was performed using $1 \%$ formaldehyde during $20 \mathrm{~min}$ incubation on ice. Finally, the cells were washed once in $1 \mathrm{ml} 1 \%$ BSA after which the pellets were resuspended in $500 \mu \mathrm{l}$ of PBS and were subjected to flow cytometry acquisition using Attune NxT (Thermofisher Scientific) instrument. The expression levels of the examined cell surface markers were acquired using FlowJo software.

For flow cytometric analysis, primary human AML cells were washed and suspended in PBS with 1\% FBS. Cells were then incubated with a viability reagent, viakrome 808 (Beckman coulter) for $20 \mathrm{~min}$ at $4{ }^{\circ} \mathrm{C}$. Then cells washed and centrifuged at $500 \mathrm{x}$ g and stained with fluorescence-labelled antibodies against CD14-FITC, CD11b-APC-Cy7, (Biolegend ${ }^{\circledR}$, San Diego, CA, USA). Flow cytometry experiments were performed on a Beckman Coulter CytoFLEX LX and all data were analyzed with FlowJo software (V10.0.7, TreeStar, Ashland, OR, USA).

\section{Lentiviral transduction of murine leukemic cells}

Lentiviral constructs (3 different shRNAs targeting Smarca5) were transiently transfected in HEK-293 T cells by using the calcium phosphate transfection method together with envelope plasmid $p V S V-G$, and packaging plasmids $\mathrm{p} M D L$ and $p R E V$. Viral supernatants were collected upon $48 \mathrm{~h}$ of transfection and filtered using $0.45 \mu \mathrm{m}$ filter. The transduction of $5 \times 10^{5}$ NUP98NSD1/FLT3-ITD cells was performed in $2 \mathrm{ml}$ volume (in 6 well plate) comprising viral supernatants mixed with RPMI 1640 supplemented with $20 \mathrm{ng} / \mathrm{ml}$ SCF, $10 \mathrm{ng} /$ $\mathrm{ml} \mathrm{IL-3,} \mathrm{and} 5 \mathrm{ng} / \mathrm{ml} \mathrm{IL-} 6$ and polybrene $(8 \mu \mathrm{g} / \mathrm{ml})$. The plates were centrifuged for $90 \mathrm{~min}$ at $2500 \mathrm{rpm}$ at $30^{\circ} \mathrm{C}$. Upon centrifugation, the cells were incubated for $4 \mathrm{~h}$, after which $2 \mathrm{ml}$ of RPMI 1640 medium supplemented with IL-3, IL-6, and SCF was added. Day after, the medium was refreshed and the cells were subjected to puromycin antibiotic selection using the concentration of $2 \mu \mathrm{g} /$. Upon 4 days, selection medium was removed, and cells that survived were expanded. For the cloning of SMARCA5 shRNAs we used TRCN0000295773 and TRCN0000288446 forward and reverse oligonucleotides, while for the BPTF shRNAs TRCN0000238661 and TRCN0000238664 oligonucleotides were utilized (RNAi Consortium, Broad Institute). 
SMARCA5 knock-down and colony-formation assay

For the induction of Smarca5 knock-down, shRNA transduced cells were plated in RPMI1640 medium supplemented with IL-3, IL-6, SCF, and doxycycline $(1 \mu \mathrm{g} /$ $\mathrm{mL}$ ). Fresh medium containing doxycycline was continuously exchanged after 48-h-cycles. For colony-forming assays, $15 \times 10^{3}$ shRNA transduced and non-transduced NUP98-NSD1/FLT3-ITD cells were plated in $1.5 \mathrm{~mL}$ of methylcellulose (MethoCult M3434, StemCell Technologies) containing IL-3, IL-6, SCF and were grown with/ without doxycycline $(1 \mu \mathrm{g} / \mathrm{mL})$. Colonies were scored microscopically after 10 days.

\section{May-Grunwald Giemsa staining}

NUP98-NSD1/FLT3-ITD transduced murine leukemic cells were counted and $2 \times 10^{5}$ cells were spinned at $250 \mathrm{rpm}$ for $5 \mathrm{~min}$. Cytospin slides were dried overnight before the staining procedure. The cells were stained for $8 \mathrm{~min}$ with May-Grunwald solution, washed 3 times in deionized water and then incubated for $40 \mathrm{~min}$ with Giemsa staining solution. After three more washes with water, samples were dried overnight and the images were acquired using Olympus BX63 upright microscope equipped with Leica DFC450C color camera.

\section{Results}

NUP98-NSD1 interacts with SMARCA5 and binds its cancer co-dependent partners BPTF and NUP188

Due to the absence of patient-derived NUP98-NSD1 ${ }^{+}$ AML cell lines and limited access to primary tumor cells we determined protein-protein interactions of NUP98NSD1 in human embryonic kidney (HEK-293) cells. We transiently expressed FLAG-NUP98-NSD1 (control cells were transfected with empty FLAG vector) and prepared nuclear lysates for immunoprecipitation (IP) followed by mass spectrometry. Protein label-free quantification (LFQ) values from two replicate samples were analyzed using two-samples t-test statistics (Benjamini-Hochberg correction with $\mathrm{FDR}=0.05$ ) leading to the quantification of 502 proteins out of which 182 were statistically significant potential NUP98-NSD1 interactors (Supplementary Table 1). Importantly, the previously validated NUP98-NSD1 interactors RAE1 and XPO5 [19] were among the most significantly enriched proteins (Fig. 1A). Another previously known interactor, the EP300 histone acetyl-transferase [20], was validated by Westernblotting (Fig. 1B). In addition, the interactions with SMARCA5, BPTF, ADNP, KDM1A, CHD4 and POL2A were also verified by Western-blotting (Fig. 1B). To get mechanistic insights, potential functional co-operation among top 20 most enriched interactions was examined using the Cancer Dependency Map (Depmap) which revealed that SMARCA5, BPTF and NUP188 operate as a co-dependent functional unit in cancer cells (Fig. 1C). To determine the potential functional relations between NUP98-NSD1 and SMARCA5 we examined their genomic binding in leukemic cells using public ChIP-Seq datasets. Peak enrichment analysis showed that NUP98NSD1 co-localized with SMARCA5 at $65 \%$ of its target sites. Moreover, Gene Set Enrichment Analysis (GSEA) revealed that co-bound genes are involved in regulation of stem cell differentiation and positive regulation of transcription from RNA II polymerase promoter (Fig. 1D). Finally, a separate analysis of the interactomes of WT NSD1 and the NUP98 N-terminus uncovered that SMARCA5 was the only protein among the 20 most enriched interactors, to be bound independently by WTNSD1 and N-terminal NUP98, which suggests a deeply rooted molecular connection with the fusion protein (Supplementary Fig. 1 \& Tables 2 and 3). Taken together, systematic analysis of NUP98-NSD1-bound proteome identified the ISWI family protein SMARCA5 (and its cancer co-dependent partners BPTF and NUP188) as stable interactors and revealed their potential functional co-operation in transcriptional regulation of stem cell differentiation.

\section{NUP98-NSD1 core interactome binding relies on NUP98 FG repeat domains}

To examine the role of different NUP98-NSD1 domains in interactome organization, we generated NUP98-NSD1 mutants bearing deletions of one or both FG repeat motifs (FLAG- $\triangle$ NUP98FG1-NSD1, FLAG- NUP98FG2-

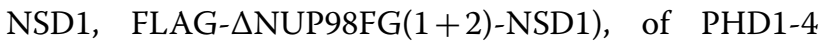
domains (FLAG-NUP98- $\triangle$ NSD1PHD1-4), and of PHD5 and $\mathrm{C} 5 \mathrm{HCH}$ domains (FLAG-NUP98- $\triangle$ NSD1PHD5$\mathrm{C} 5 \mathrm{HCH}$ ) and transiently expressed them in HEK-293 cells (Fig. 2A). We achieved almost equilibrated expression and immunoprecipitation of the mutated constructs (Fig. 2D, Supplementary Fig. 2). Interactors of full-length NUP98-NSD1 and of its mutated versions were identified by label free quantitative proteomics. Strikingly, principal component analysis (PCA) separated the interactomes acquired for the FG-repeat motif deleted fusions and control on the one side, and full-length and PHD1-4 and PHD5-C5HCH deleted fusions on the other side, suggesting a prominent role of the disordered domains in the interactome organization (Fig. 2B-D, Supplementary Fig. 3). To assess the impact of the mutated domains on the core interactome, we analyzed the LFQ values of the top 20 NUP98-NSD1 interactors and observed significant perturbations in this core interactome upon deletions of single or both FG repeat domains, whereas deletions of the PHD domains barely affected these interactions (Fig. 2C, Supplementary Fig. 3, Supplementary Tables 4-6). In particular, SMARCA5, BPTF and NUP188 


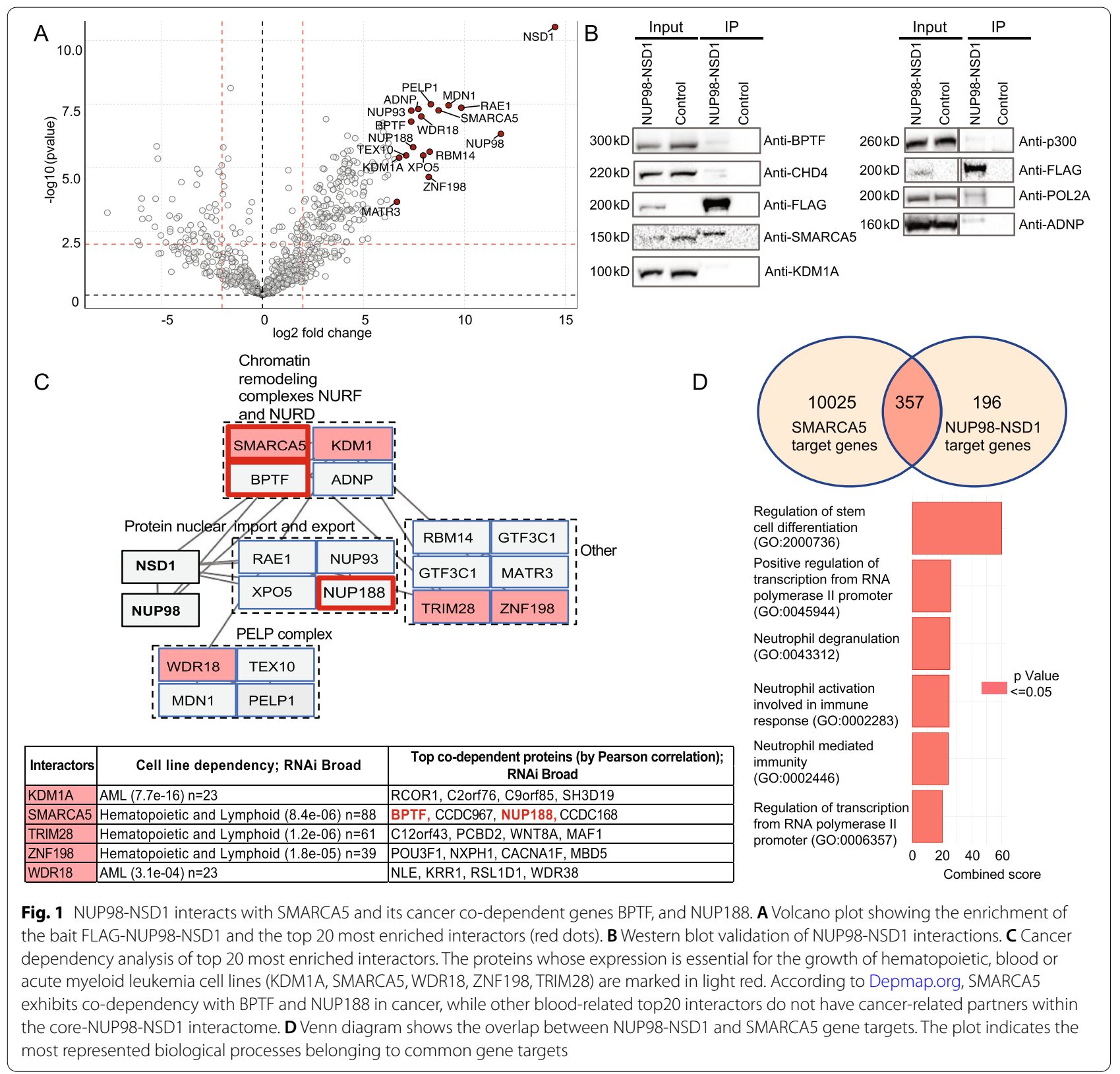

were lost upon deletion of one or both FG repeat domains. In addition, interactions with KDM1, NUP93, XPO5, RBM14, or MDN1 were also evicted in absence of one or both FG repeats (Fig. 2C). As expected, the interaction with RAE1, mediated by the GLEBS domain of NUP98 moiety, was not affected by FG repeat deletions (Fig. 2C, Supplementary Fig. 3B), indicating that our analysis identified specific, FG-repeat mediated interactions. Loss of interaction with BPTF, SMARCA5, KDM1A, CHD4 and POL2A was confirmed by Western blotting (Fig. 2D). Taken together, mutational studies revealed a prevalent role of the NUP98 FG repeat domains in mediating the interactome network of NUP98-NSD1.

\section{NUP98 FG repeat domains mediate the formation of NUP98-NSD1 liquid-like phase separated nuclear condensates}

To examine the nuclear localization pattern of NUP98NSD1 and the dependency on its structural domains, we performed immunofluorescence staining of transiently expressed full-length and mutated NUP98NSD1 constructs. Confocal imaging showed that the 


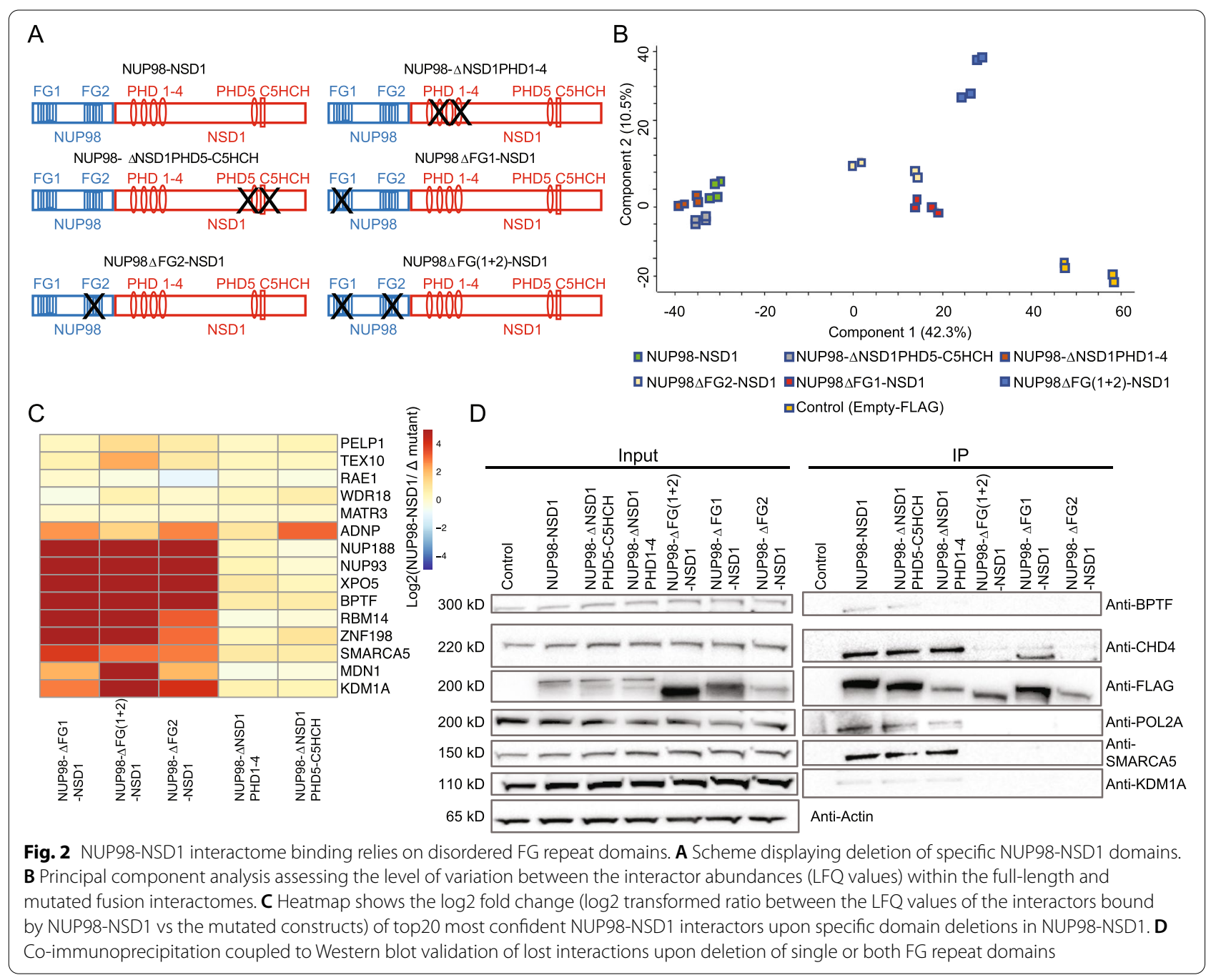

full-length NUP98-NSD1 displayed a "nuclear puncta" distribution, dependent on both FG-repeat domains as their deletion led to complete dispersion and to a more peripheral localization of the fusion protein (Fig. 3A). On the other side, deletion of PHD1-4 and PHD5$\mathrm{C} 5 \mathrm{HCH}$ domains did not significantly affect the formation of nuclear puncta, suggesting that FG repeat domains are pivotal for the correct nuclear localization of NUP98-NSD1 (Fig. 3A). As these disordered domains mediate phase separation of the full-length NUP98 within the NPC [21], we examined whether NUP98-NSD1 exhibits phase-separating properties. To assess the phase-transition potential of NUP98-NSD1 in vitro, we tested its ability to precipitate in presence of biotinylated isoxazole, a compound that induces the precipitation of proteins with disordered domains [18]. While the full-length protein and the PHD-deleted forms achieved complete precipitation with $30 \mu \mathrm{M}$ b-isoxazole, the FG-deleted constructs exhibited a "leakage" in the unprecipitated fraction (Fig. 3B), suggesting that FG repeat domains are indeed required for phase transition of NUP98-NSD1. In addition, NUP98NSD1 nuclear puncta were sensitive to 1,6 hexanediol treatment, a compound that disrupts the formation of liquid-liquid like biomolecular condensates [22], thus confirming their phase-separated nature (Fig. 3C). To analyze the properties of NUP98-NSD1 nuclear condensates in vivo, we transiently expressed a NUP98NSD1-GFP fusion and performed FRAP analysis to study the dynamics of the protein in live cells. As positive control, HEK293 cells were transfected with GFPBP53, known to exhibit liquid-like phase separation in live cells [23]. After photobleaching, NUP98-NSD1-GFP nuclear puncta recovered fluorescence on a time-scale of $\sim 50 \mathrm{~s}$, and exhibited significantly faster motility than GFP-BP53 which recovered fluorescence after $\sim 90 \mathrm{~s}$, 


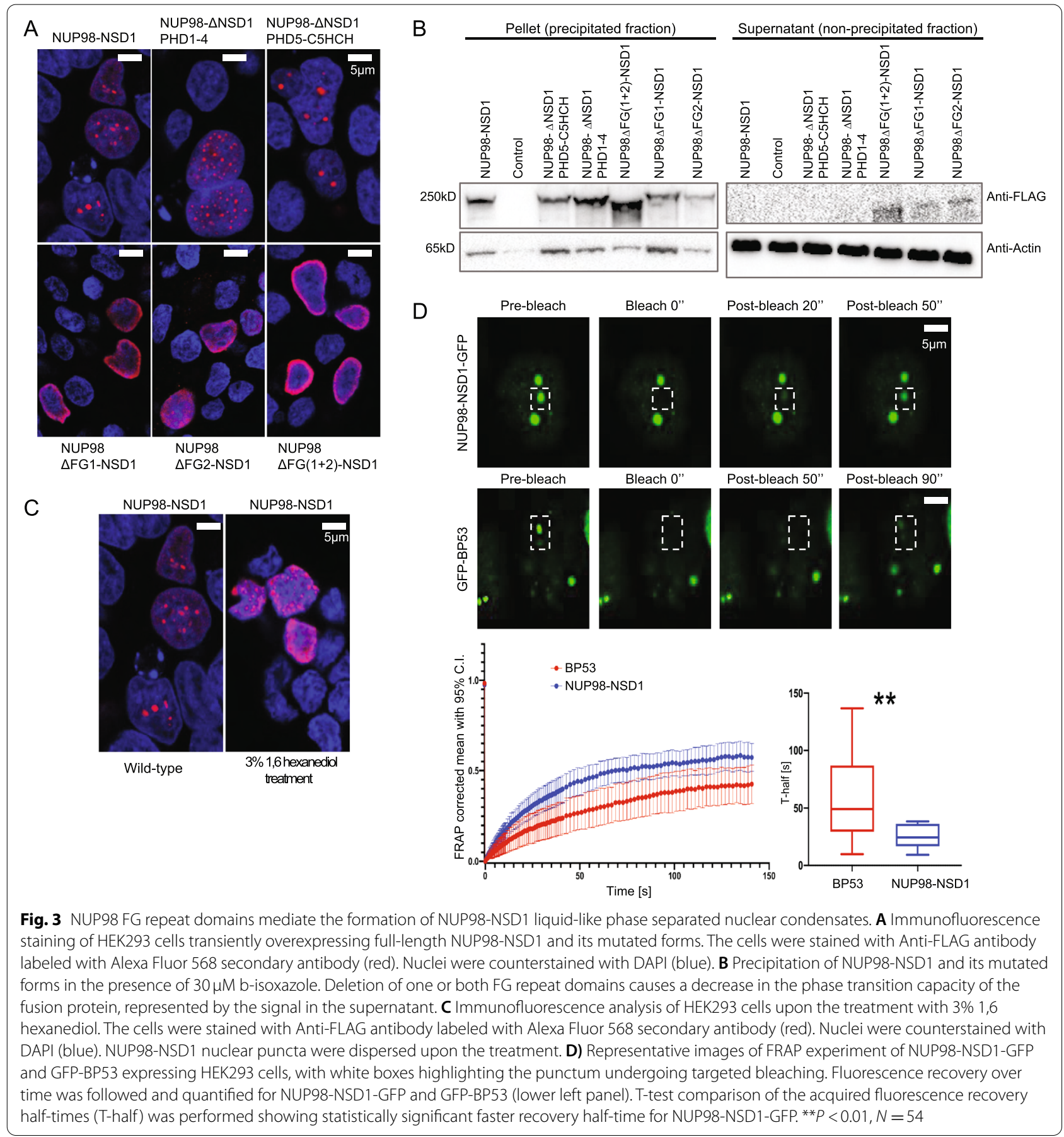

thus demonstrating the liquid-like nature of phase-separated NUP98-NSD1 nuclear puncta (Fig. 3D). Taken together, our data suggest that NUP98-NSD1 forms liquid-like phase-separated nuclear condensates dependent on NUP98 FG repeat domains. Moreover, in line with proteomics data, the identified FG-repeat mediated interactors are bona fide constituents of NUP98NSD1 condensates.

\section{NUP98-NSD1 interactions and condensate formation are conserved in NUP98-NSD1/FLT3-ITD immortalized hematopoietic cells}

To assess whether the phase-separated nature of NUP98-NSD1 is conserved in hematopoietic cells, we expressed the full-length NUP98-NSD1 and $\triangle \mathrm{NUP98FG}(1+2)-\mathrm{NSD} 1$ in $32 \mathrm{Dcl} 3$ murine myeloid progenitor cells. Importantly, the fusion protein kept the 
nuclear puncta localization pattern, which was dependent on the presence of FG repeats (Supplementary Fig. 4). To study the functional impact of our findings on NUP98-NSD1-mediated transformation, we immortalized primary murine bone marrow cells by retroviral expression of NUP98-NSD1 and FLT3-ITD, previously shown to be necessary for efficient immortalization of primary murine hematopoietic stem and progenitor cells [24]. After expansion under antibiotic selection and enrichment by GFP sorting (Supplementary Fig. 5A), we confirmed the presence of NUP98-NSD1 nuclear puncta by immunofluorescence staining (Fig. 4A). GiemsaWright staining revealed a blast-like morphology of NUP98-NSD1/FLT3-ITD cells (Supplementary Fig. 5B). To examine whether the fusion protein interacts with SMARCA5 and BPTF also in these cells, we performed proximity ligation assays. We found that the signal for both interactions was specifically located in the nucleus of NUP98-NSD1/FLT3-ITD cells, while control samples showed low background (Fig. 4B). To address the clinical relevance of our findings, we examined nuclear localization pattern and tested the observed interactions in NUP98-NSD1 ${ }^{+}$patient cells, using the antibodies targeting N-terminal NUP98 and C-terminal NSD1. Immunofluorescence staining of both moieties showed punctual pattern of nuclear localization (Fig. 4C), which was in line with recently published findings in patient cells expressing NUP98-HOXA9, NUP98- PRRX1, NUP98-KDM5A, and NUP98-LNP1 fusions [5]. Moreover, we detected the interaction between SMARCA5 and both fusion moieties in NUP98-NSD $1^{+}$patient cells, thus confirming the existence of this interaction in physiological conditions (Fig. 4D). Overall, these data indicate that the NUP98NSD1 interactome and nuclear localization identified in HEK-293 cells are present in NUP98-NSD1 expressing patient cells and bona fide maintained in immortalized primary murine hematopoietic cells.

\section{SMARCA5 is indispensable for NUP98-NSD1-mediated transformation and expression of the fusion target genes} To examine the functional role of SMARCA5 and BPTF interactors in NUP98-NSD1/FLT3-ITD immortalized hematopoietic cells, we performed doxycycline-inducible (DOX) knock-down of both nuclear condensate constituents. We confirmed the knockdown of SMARCA5 at protein and of BPTF at the mRNA level (Fig. 5A, B), and analyzed the clonogenic growth of NUP98-NSD1/FLT3ITD transformed cells in methylcellulose. Although SMARCA5 and BPTF proteins cooperate within the NURF chromatin remodeling complex, the transformed cells were specifically sensitive to SMARCA5 loss (Fig. 5C), which completely abolished colony formation (Fig. 5D) thus indicating its functional role in mediating NUP98-NSD1 transformation. Interestingly, the knockdown of SMARCA5 did not influence formation of fusion phase separated nuclear condensates (Fig. 5E), despite recently reported critical role of biomolecular condensation in NUP98 leukemogenesis, suggesting the importance of fully functional interactome network in the maintenance of NUP98-NSD1 transformation. To further examine SMARCA5 function in transformed cells, we performed treatment with an allosteric inhibitor (ED2-AD101) targeting the ATPase activity of the ISWI family of chromatin remodelers (Supplementary Fig. 5) $[25,26]$, which consistently prevented colony formation in methylcellulose (Supplementary Fig. 6). NUP98-NSD1 requires catalytically active SMARCA5 for the maintenance of hematopoietic transformation, as the interaction remained unaffected upon pharmacological inhibition (Fig. 5F). Finally, SMARCA5 genetic and pharmacological targeting led to reduced expression of NUP98-NSD1 gene targets HoxA9 and Meis1, which proposes its role in regulation NUP98-NSD1-driven transcriptional program (Fig. 5G). To further address the clinical relevance of ED2-AD101, we treated NUP98-NSD1 ${ }^{+}$patient cells using $10 \mu \mathrm{M}$ concentration (following the previously calculated $\mathrm{IC}_{50}$ in transformed murine hematopoietic cells, Supplementary Fig. 6) and found some increase in expression of the CD11b and CD14 surface markers of myeloid differentiation (Supplementary Fig. 7A). However, due to high cell death (Supplementary Fig. 7B) upon inhibitor treatment we could not characterize the exact effect of the inhibitor, probably caused by the low specificity of the compound. In summary, our genetic and

\footnotetext{
(See figure on next page.)

Fig. 4 NUP98-NSD1 retains nuclear localization pattern and interactions in murine NUP98-NSD1/FLT3-ITD immortalized hematopoietic stem and progenitor cells and in NUP98-NSD1 ${ }^{+}$patient cells. A Immunofluorescence staining showing the nuclear puncta pattern of localization of the fusion in primary murine NUP98-NSD1/FLT3-ITD immortalized hematopoietic cells. The cells were stained with Anti-FLAG antibody labeled with Alexa Fluor 568 secondary antibody (green). Nuclei were counterstained with DAPI (blue). B Proximity ligation assay in NUP98-NSD1/FLT3-ITD immortalized hematopoietic cells showing the interaction between NUP98-NSD1 and SMARCA5/BPTF, detected using kit specific fluorophore (lem $=669 \mathrm{~nm}$, far red). Nuclei were counterstained with DAPI (blue). C Immunofluorescence staining showing the nuclear puncta pattern of localization of the fusion in NUP98-NSD ${ }^{+}$patient cells. The cells were stained with Anti-NSD1 and Anti-NUP98 antibodies targeting the C-terminus and $\mathrm{N}$-terminus (epitopes included in the fusion protein), respectively. Primary antibodies were then labeled with Alexa Fluor 647 secondary antibody (green). Nuclei were counterstained with DAPI (blue). D Proximity ligation assay in NUP98-NSD1+ patient cells showing the interaction between the N-terminal NUP98 and C-terminal NSD1 moieties of the fusion with SMARCA5, detected using kit specific fluorophore (lem $=669 \mathrm{~nm}$, far red), on the right. Nuclei were counterstained with DAPI (blue)
} 

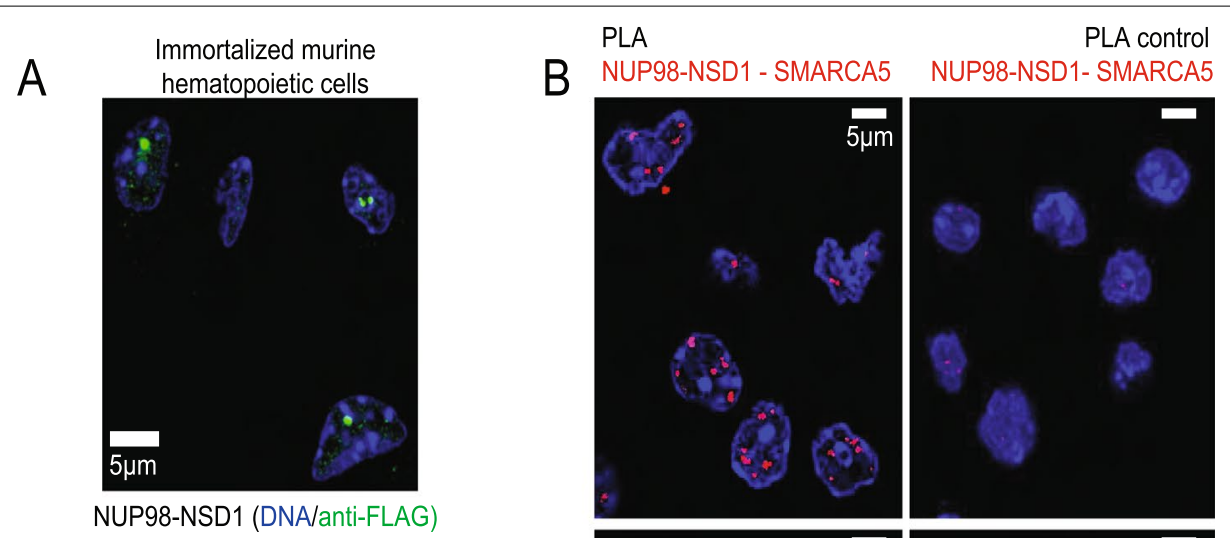

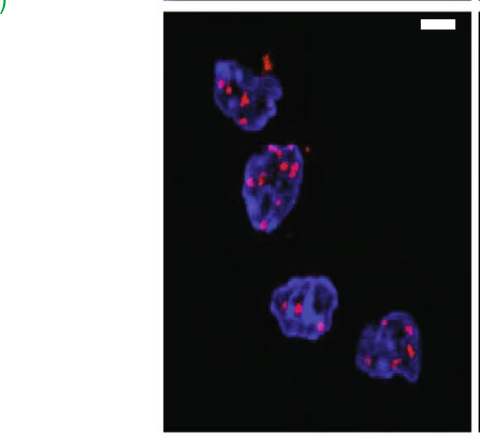

PLA

D

NUP98-NSD1 - BPTF

PLA

NSD1 (C-term) - SMARCA5
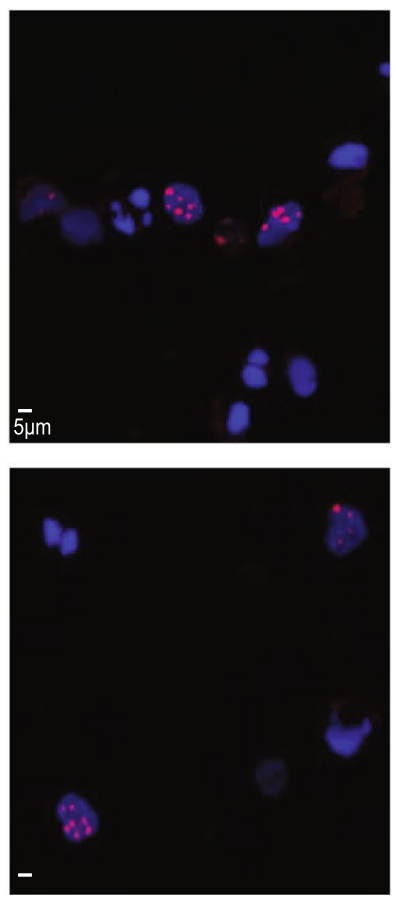

PLA

NUP98 (N-term) - SMARCA5

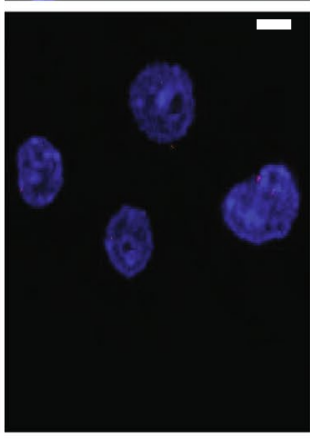

PLA control NUP98-NSD1 - BPTF

PLA control NSD1 (C-term) - SMARCA5
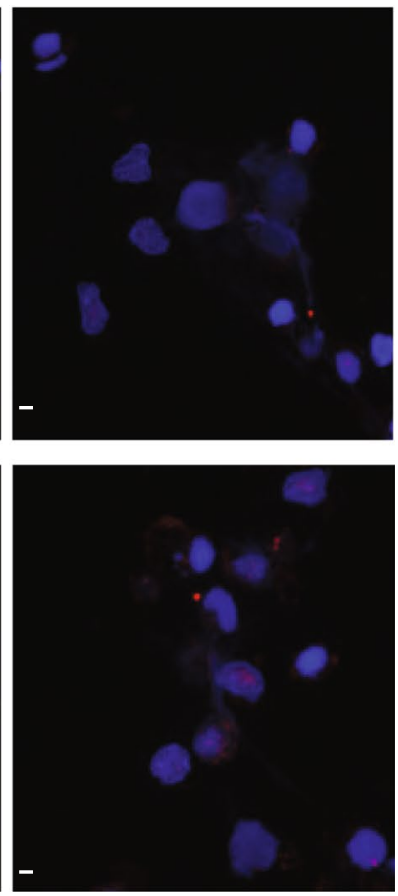

PLA control NUP98 (N-term) - SMARCA5

Fig. 4 (See legend on previous page.) 
pharmacologic data in transformed murine and patient cells suggest a pivotal role of SMARCA5, constituent of the NUP98-NSD1 nuclear condensates, in the maintenance of NUP98-NSD1-mediated transformation.

\section{Discussion}

We characterized the nuclear core interactome of the AML-associated NUP98-NSD1 fusion protein and identified the constituents of the NUP98 phenylalanine-glycine (FG) repeat-dependent nuclear condensates. We also found functional co-dependencies of condensate constituents including the transcriptional coregulators SMARCA5 and BTPF. Genetic and pharmacological interference characterized SMARCA5 as NUP98-NSD1 interacting protein critical for aberrant clonogenic growth of NUP98-NSD1 immortalized primary murine hematopoietic cells. Notably the interaction of NUP98NSD1 and SMARCA 5 was also found in primary patientderived AML cells carrying this fusion.

Several very recent studies proposed that FG repeatmediated biomolecular condensation of NUP98 fusions is crucial and sufficient to activate a leukemogenic transcriptional program [4-6]. However, none of the studies examined any molecular players that cooperate with NUP98 fusions in installing the leukemogenic gene regulatory network.

In our study, we demonstrated that both genetic and pharmacological targeting of SMARCA5 did not affect the condensates indicating that biomolecular condensation per se is not sufficient to maintain the transformed phenotype. One recent study compared the protein interactome of five AML-associated epitope-tagged NUP98 fusions, including NUP98-NSD1, ectopically expressed in the human HL-60 AML cell line and identified by mass spectrometry 157 shared interactors [6]. Notably, in contrast to our study, Terlecki-Zaniewicz and colleagues identified SMARCA5 (also known as SNF2H) as general constituent of biomolecular condensates in human HL-60 AML cells, but not as interactor of any of the 5 NUP98-fusions studied. We believe that the reason for the observed discrepancy lies in diverse experimental approaches linked to different objectives of this recent publication. Terlecki-Zaniewicz and colleagues performed a very challenging screen for common interactions among 5 different NUP98 fusions using whole cell lysates. On the other hand, we used nuclear extracts in our mass spectrometry and Western blot analysis (as NUP98-NSD1 is expressed exclusively in the nucleus), in order to reduce the number of cytoplasmic contaminants. Furthermore, we focused on dissecting in-depth the nuclear interactome of solely NUP98-NSD1, and therefore utilized an immunoprecipitation protocol enabling the detection of high-confidence protein-protein interactions [27]. Moreover, we provided specific functional information related to the dissection of NUP98-NSD1 nuclear condensate constituents through structural domain-dependent interactome analysis. Finally, we also validated some interactions in NUP98-NSD1 immortalized primary murine hematopoietic cells and patient cells using proximity ligation assay.

Another recent study demonstrated that altered chromatin looping, induced by the phase separation of NUP98-HOXA9, is driving the onset of the leukemogenic transcription programs [4]. Protein-mediated phase separation contributes to the three-dimensional organization of the genome and transcription regulation, thus influencing the expression of gene regulatory networks driving the cell fate [28]. SMARCA5 chromatin remodeler is one of the principal regulators of genome-wide nuclear topology, and is deeply involved in recruitment of transcription factors, such as CTCF [29]. We showed that genomic co-localization of SMARCA5 and NUP98NSD1 occurs at the transcriptionally active genomics sites regulating stem cell differentiation, therefore suggesting that SMARCA5 may recruit NUP98-NSD1 to its target sites, thus promoting leukemic transformation.

Genetic ablation of Smarca5 in mice leads to an early lethal phenotype due to blocked maturation of erythroid and myeloid lineages underlining its role as a critical hematopoietic regulator [30]. In hematopoiesis, highest

\footnotetext{
(See figure on next page.)

Fig. 5 SMARCA5 genetic and pharmacologic targeting reduce self-renewal of NUP98-NSD1/FLT3-ITD immortalized hematopoietic stem and progenitor cells and downregulate HoxA9 and Meis1 expression. A Western-blot analysis showing the expression of SMARCA5 in NUP98-NSD1/ FLT3-ITD hematopoietic cells. B RT-qPCR analysis showing the knockdown of BPTF at mRNA level, upon 1st and 2nd methylcellulose (re)plating using 2 different primers for 2 different shRNAs. C Colony numbers after (re)plating in methylcellulose upon treatment with $2 \mu \mathrm{g} / \mathrm{ml}$ doxycycline. $N=3$ biological replicates. T-test, ${ }^{* * *} P<0.0005$. Data are mean \pm SD. $\mathbf{D}$ Colony formation capacity in methylcellulose after plating in presence of $2 \mu \mathrm{g} / \mathrm{ml}$ doxycycline. The images represent the effect observed in 3 biological replicates. E Immunofluorescence staining of NUP98-NSD1/ FLT3-ITD immortalized hematopoietic cells upon doxycycline induced knockdown of SMARCA5, showing formation of the fusion's nuclear condensates irrespectively from SMARCA5 expression. The cells were stained using Anti-FLAG and Anti-SMARCA5 antibodies, labeled with Alexa Fluor 568 secondary antibody (red), and Alexa Fluor 647 secondary antibodies (green), while nuclei were counterstained with DAPI (blue). F Proximity ligation assay in NUP98-NSD1/FLT3-ITD immortalized hematopoietic cells showing the lack of the signal for the interaction between NUP98-NSD1 and SMARCA5 in shRNA1 + DOX (SMARCA5 knockdown) condition and upon treatment with $10 \mu$ M ED2-AD101. G HoxA9 and Meis1 expression measured by RT-qPCR in cells upon induced shRNA-mediated knockdown of SMARCA5 $(1 \mu \mathrm{g} / \mathrm{ml}$ doxycycline) and treatment with $10 \mu \mathrm{M}$ ED2-AD101. $N=3$ biological replicates. T-test, ${ }^{*} P<0.05$. Data are mean \pm SD
} 

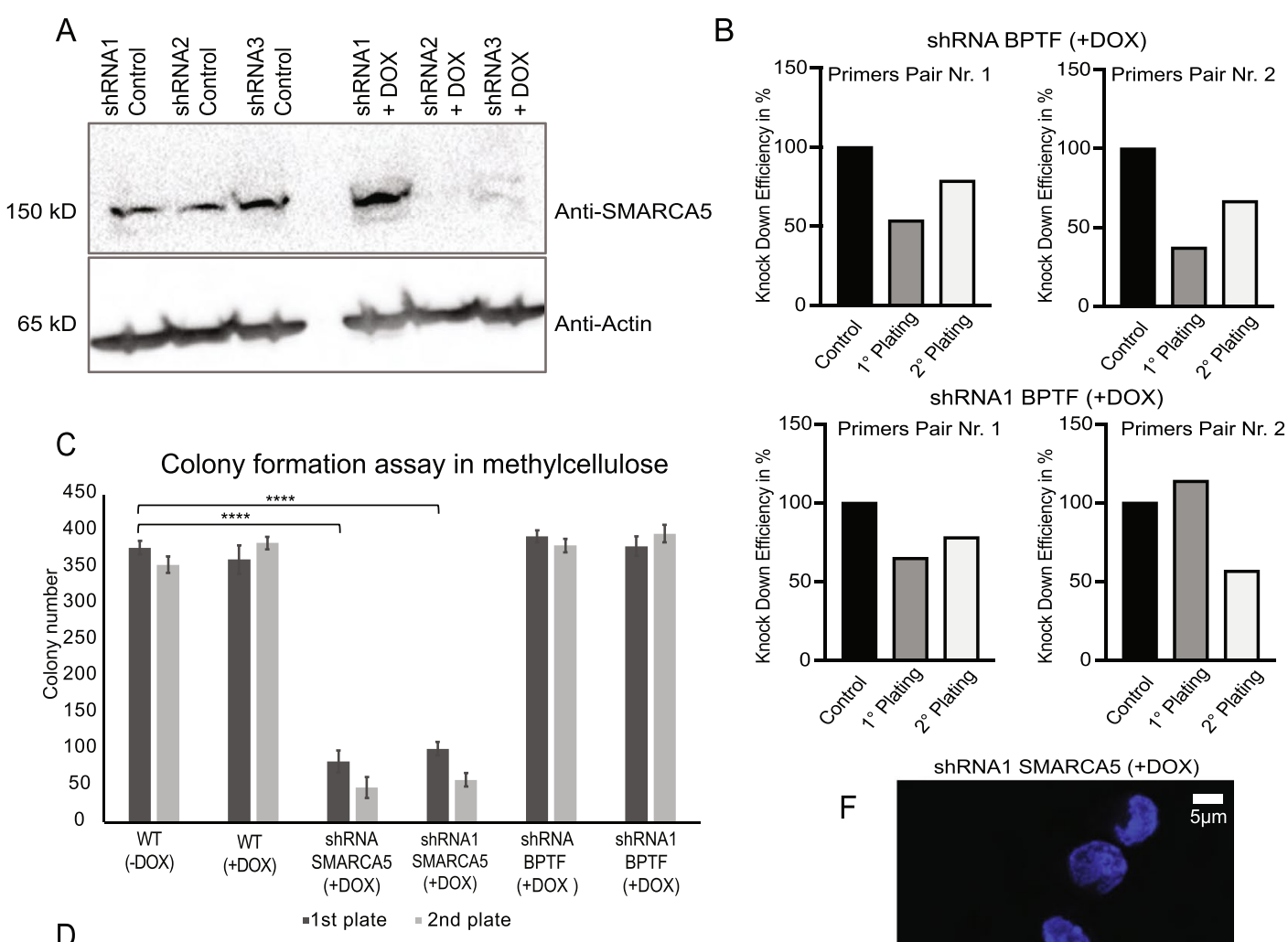

ShRNA SMARCA5 (-DOX) ShRNA SMARCA5 (+DOX) ShRNA1 SMARCA5 (+DOX)

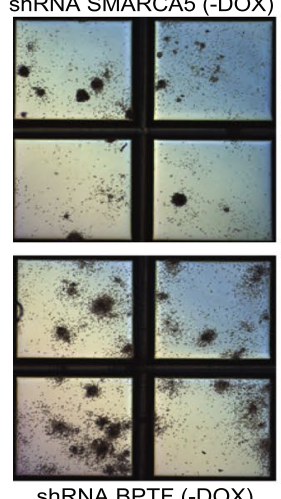

shRNA BPTF (-DOX)

E

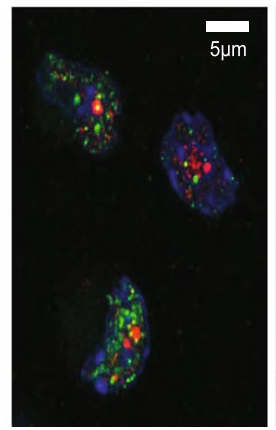

SMARCA5

NUP98-NSD

Fig. 5 (See legend on previous page)
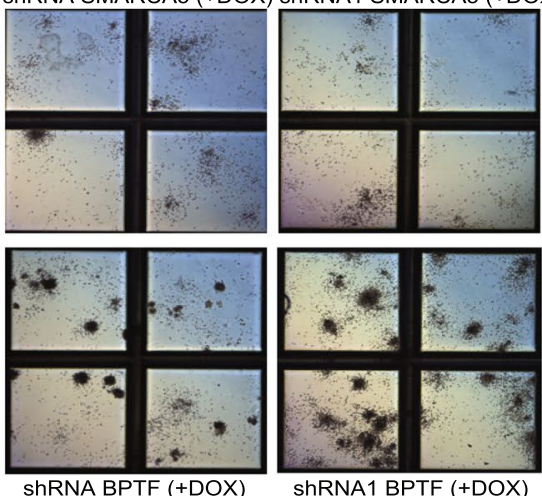

ShRNA (+DOX)

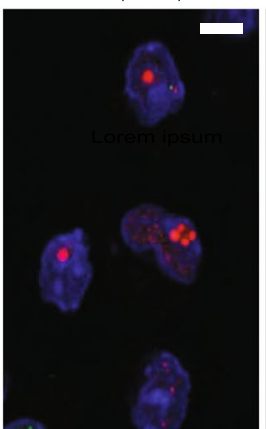

SMARCA5

NUP98-NSD1

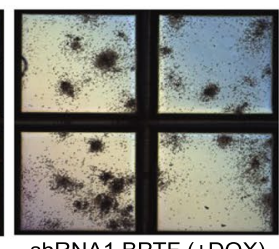

shRNA1 BPTF (+DOX)

ShRNA1 (+DOX)

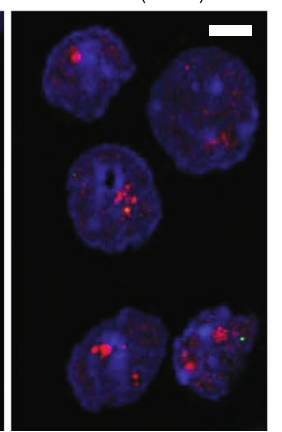

SMARCA5

NUP98-NSD1
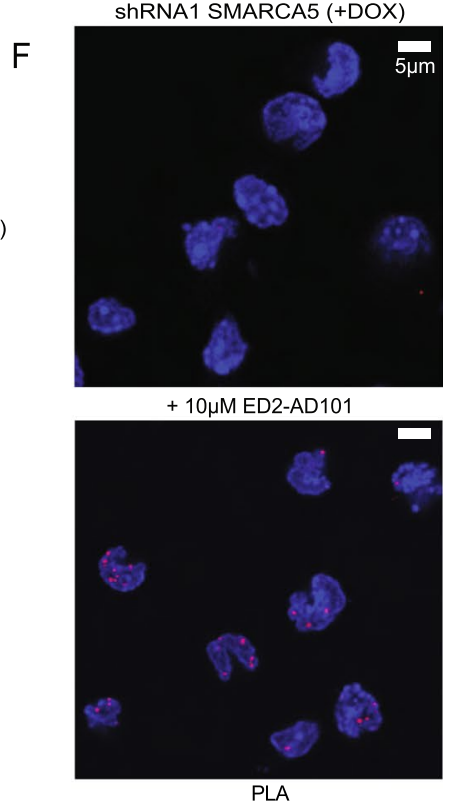

NUP98-NSD1 - SMARCA5

G RT-qPCR for HoxA9 and Meis1

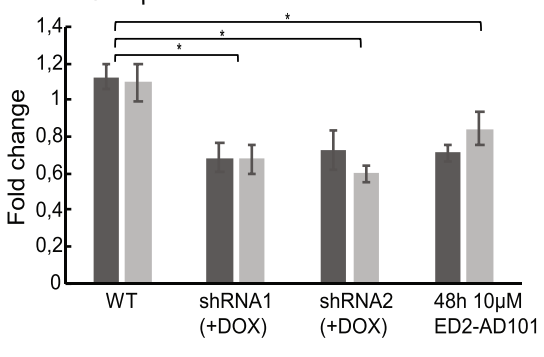

- HoxA9 $=$ Meis1 
SMARCA5 levels are expressed in progenitor cells of the myeloid and erythroid lineage (http://servers.binf.ku.dk/ bloodspot/). Likewise, exposure to a small molecule inhibitor (ED2-AD101) targeting the chromatin remodelers SMARCA5 and CHD4 at doses of between 1 to $10 \mu \mathrm{M}$ induced differentiation of THP-1 leukemic cell line [25]. We observed some degree of differentiation of NUP98$\mathrm{NSD}^{+}{ }^{+}$patient cells upon treatment with $10 \mu \mathrm{M}$ ED2AD101, and very high rate of cell death, most probably due to the low specificity of the compound. Therefore, more selective SMARCA5-inhibiting compounds are clearly necessary to explore a therapeutic window against AML.

We, as well as Terlecki-Zaniewicz and colleagues, did not identify the previously published interaction of NUP98-fusions with KMT2A detected using whole-cell lysates and proximity dependent biotinylation (BioID) followed by Western blotting [11]. This may be explained by the biochemical complementarity of the different approaches [31, 32]. We also compared our NUP98-NSD1 interactome with the affinity-purified NUP98-HOXA9 interactome reported by Shima et al. and found a rather low overlap, suggesting that two fusion proteins may utilize different mechanisms for their molecular function [33]. Nevertheless, both datasets shared previously published NUP98 interactions with RAE1 and XPO1.

\section{Conclusions}

In conclusion, we characterized multiple nuclear protein interactions of the AML-associated NUP98-NSD1 fusion and identified SMARCA5 as important mediator to maintain the transformed phenotype of NUP98-NSD1immortalized hematopoietic cells. Our data contain thorough functional information that may be crucial for designing specific inhibitors to be used in NUP98-NSD1 driven AML.

\footnotetext{
Abbreviations

AML: Acute myeloid leukemia; FG: Phenylalanine-glycine; ISWI: Imitation switch family of proteins; BPTF: Bromodomain PHD finger transcription factor; NURF: Nucleosome remodeling factor; NUP98: Nucleoporin 98; NSD1: Nuclear receptor SET domain; PHD: Plant homeodomain; $\mathrm{C} 5 \mathrm{HCH}$ : Cys-His-rich; PWWP: Proline-tryptophan-proline; ATCC: American Type Culture Collection; TFA: Trifluoroacetic acid; PLA: Proximity ligation assay; FRAP: Fluorescent recovery after photobleaching; b-isox: b-isoxazole; 5-FU: 5-Fluorouracil; IL-3: Interleukin 3; IL-6: Interleukin 6; SCF: Stem cell factor; HEK293: Human embryonic kidney cells; IP: Immunoprecipitation; LFQ: Label-free quantification; GSEA: Gene set enrichment analysis; PCA: Principal component analysis; DOX: Doxycycline; shRNA: Small hairpin RNA; BiolD: Proximity dependent biotinylation.
}

\section{Supplementary Information}

The online version contains supplementary material available at https://doi. org/10.1186/s13046-022-02248-X.

Additional file 1.

\section{Acknowledgments}

We thank IFOM Functional Proteomics group and Proteomics facility for critical comments and suggestions. We thank IFOM Imaging technological development unit for their assistance in immunofluorescence imaging and sorting (Maria Grazia Totaro and Sara Martone) experiments. We thank Fabio lannelli and Federica Zanardi from IFOM Bioinformatics unit for their precious help with data analysis. We thank Professor Juri Rappsilber (TU Berlin) for comments and suggestions regarding AP-MS experiments. We thank Professor Diego Pasini (IEO, Milan) for insightful discussions. We thank Professor Aniruddha Deshpande (Sanford Burnham Prebys Medical Discovery Institute, San Diego) for FLAG-NUP98-NSD1 construct. We thank Professor Yogen Saunthararajah from Cleveland Hospital for providing ED2-AD101. We thank Professor Robert Slany (University Erlangen-Nürnber) for precious discussion. We thank Sabine Juge-Ehret and Federica Valigi from Childhood Leukemia group in Basel for help with primary murine hematopoietic progenitors.

\section{Authors' contributions}

Z.J. designed the study, performed all the experiments and analyzed the results. V.M. mentored immunoprecipitation experiments, mass spectrometry acquisitions and statistical analysis. F.C. performed confocal imaging, M.G. performed FRAP measurements. F.S. mentored methylcellulose experiments, Giemsa-Right staining. J.S. mentored immortalization assay. M.R. performed the experiments with patient cells. O.H. provided the access to patient cells. Z.J., J.S., A.B. wrote the original draft. A.B., J.S., S.M., O.H, G.M. provided resources and ideas. A.B. supervised the overall study and provided funding. The authors read and approved the final manuscript.

\section{Funding}

This work was supported by the Italian Ministry of Health (RF-2013-02354880) and AIRC IG 18607. The work in the laboratory of J.S. was supported by SWISS CANCER RESEARCH (KFS-4258-08-2017).

\section{Availability of data and materials}

The mass spectrometry proteomics data have been deposited to the ProteomeXchange Consortium via the PRIDE partner repository with the dataset identifier PXD026020.

\section{Declarations}

Ethics approval and consent to participate

Not applicable.

\section{Consent for publication}

Not applicable.

\section{Competing interests}

Authors declare no competing interests.

\section{Author details}

${ }^{1}$ Functional Proteomics group, IFOM-FIRC Institute of Molecular Oncology, Milan, Italy. ${ }^{2}$ Imaging Technological Development Unit, IFOM-FIRC Institute of Molecular Oncology, Milan, Italy. ${ }^{3}$ Chromatin Alterations in Tumorigenesis Unit, European Institute of Oncology, Milan, Italy. ${ }^{4}$ Department of Biosciences, University of Milan, Milan, Italy. ${ }^{5}$ Prinses Maxima Center for Pediatric Oncology, Utrecht, The Netherlands. ${ }^{6}$ Biomolecular NMR, IRCCS Ospedale San Raffaele, Milan, Italy. ${ }^{7}$ Department for Biomedicine, University Children Hospital, Basel, Switzerland.

Received: 13 July 2021 Accepted: 10 January 2022

Published online: 24 January 2022

\section{References}

1. Khwaja A, Bjorkholm M, Gale RE, Levine RL, Jordan CT, Ehninger G, et al. Acute myeloid leukaemia. Nat Rev Dis Prim. 2016;2:16010. https://doi.org/ 10.1038/nrdp.2016.10.

2. Gough SM, Slape Cl, Aplan PD. NUP98 gene fusions and hematopoietic malignancies: common themes and new biologic insights. Blood. 
2011;118:6247 LP-6257 http://www.bloodjournal.org/content/118/24/ 6247.abstract.

3. Michmerhuizen NL, Klco JM, Mullighan CG. Mechanistic insights and potential therapeutic approaches for NUP98-rearranged hematologic malignancies. Blood. 2020;136:2275-89. https://doi.org/10.1182/blood. 2020007093.

4. Ahn JH, Davis ES, Daugird TA, Zhao S, Quiroga IY, Uryu H, et al. Phase separation drives aberrant chromatin looping and cancer development. Nature. 2021;595:591-5. https://doi.org/10.1038/s41586-021-03662-5.

5. Chandra B, Michmerhuizen NL, Shirnekhi HK, Tripathi S, Pioso BJ, Baggett DW, et al. Phase separation mediates NUP98 fusion Oncoprotein leukemic transformation. Cancer Discov. 2021; candisc.0674.2021.

6. Terlecki-Zaniewicz S, Humer T, Eder T, Schmoellerl J, Heyes E, Manhart G, et al. Biomolecular condensation of NUP98 fusion proteins drives leukemogenic gene expression. Nat Struct Mol Biol. 2021;28:190-201.

7. Hollink IHIM, van den Heuvel-Eibrink MM, Arentsen-Peters STCJM, Pratcorona M, Abbas S, Kuipers JE, et al. NUP98/NSD1 characterizes a novel poor prognostic group in acute myeloid leukemia with a distinct HOX gene expression pattern. Blood. 2011;118:3645-56. https://doi.org/10.1182/ blood-2011-04-346643.

8. Ostronoff F, Othus M, Gerbing RB, Loken MR, Raimondi SC, Hirsch BA, et al. NUP98/NSD1 and FLT3/TTD coexpression is more prevalent in younger AML patients and leads to induction failure: a COG and SWOG report. Blood. 2014;124:2400-7.

9. Jaju RJ, Fidler C, Haas OA, Strickson AJ, Watkins F, Clark K, et al. A novel gene, NSD1, is fused to NUP98 in the $t(5 ; 1)(q 35 ; p 15.5)$ in de novo childhood acute myeloid leukemia. Blood. 2001;98:1264-7.

10. Wang GG, Cai L, Pasillas MP, Kamps MP. NUP98-NSD1 links H3K36 methylation to Hox-a gene activation and leukaemogenesis. Nat Cell Biol. 2007;9:804-12 http://www.ncbi.nlm.nih.gov/pubmed/17589499.

11. Xu H, Valerio DG, Eisold ME, Sinha A, Koche RP, Hu W, et al. NUP98 fusion proteins interact with the NSL and MLL1 complexes to drive Leukemogenesis. Cancer Cell. 2016;30:863-78. https://doi.org/10.1016/j.ccell.2016. 10.019 Elsevier Inc.

12. Jordan M, Wurm F. Transfection of adherent and suspended cells by calcium phosphate. Methods. 2004;33:136-43 http://www.sciencedirect. com/science/article/pii/S1046202303003050.

13. Dignam JD, Lebovitz RM, Roeder RG. Accurate transcription initiation by RNA polymerase II in a soluble extract from isolated mammalian nuclei. Nucleic Acids Res. 1983;11:1475-89. https://doi.org/10.1093/nar/11.5. 1475.

14. Rappsilber J, Ishihama Y, Mann M. Stop and go extraction tips for matrixassisted laser desorption/ionization, nanoelectrospray, and LC/MS sample pretreatment in proteomics. Anal Chem. 2003;75:663.

15. Perez-Riverol Y, Csordas A, Bai J, Bernal-Llinares M, Hewapathirana S, Kundu DJ, et al. The PRIDE database and related tools and resources in 2019: improving support for quantification data. Nucleic Acids Res 2019;47:D442-50.

16. Cox J, Mann M. MaxQuant enables high peptide identification rates, individualized p.p.b.-range mass accuracies and proteome-wide protein quantification. Nat Biotechnol. 2008;26:1367-72 [cited 2014 Jul 10]. http://www.ncbi.nlm.nih.gov/pubmed/19029910.

17. Tyanova S, Temu T, Sinitcyn P, Carlson A, Hein MY, Geiger T, et al. The Perseus computational platform for comprehensive analysis of (prote)omics data. Nat Methods. 2016;13:731-40.

18. Kato M, Han TW, Xie S, Shi K, Du X, Wu LC, et al. Cell-free formation of RNA granules: low complexity sequence domains form dynamic fibers within hydrogels. Cell. 2012;149:753-67 https://www.ncbi.nlm.nih.gov/pubmed/ 22579281.

19. Oka M, Mura S, Yamada K, Sangel P, Hirata S, Maehara K, et al. Chromatinprebound Crm1 recruits Nup98-HoxA9 fusion to induce aberrant expression of Hox cluster genes. Elife. 2016;5:1-21.

20. Kasper LH, Brindle PK, Schnabel CA, Pritchard CEJ, Cleary ML, van Deursen JMA. CREB binding protein interacts with Nucleoporin-specific FG repeats that activate transcription and mediate NUP98-HOXA9 Oncogenicity. Mol Cell Biol. 1999;19:764 LP-776 http://mcb.asm.org/content/19/1/764.abstr act.

21. Schmidt HB, Görlich D. Nup98 FG domains from diverse species spontaneously phase-separate into particles with nuclear pore-like permselectivity. Weis K, editor. Elife, Ltd. 2015;4:e04251 eLife Sciences Publications.
22. Kroschwald S, Maharana S, Simon A. Hexanediol: a chemical probe to investigate the material properties of membrane-less compartments. Matters. 2017;3(5):e201702000010.

23. Pessina F, Giavazzi F, Yin Y, Gioia U, Vitelli V, Galbiati A, et al. Functional transcription promoters at DNA double-strand breaks mediate RNAdriven phase separation of damage-response factors. Nat Cell Biol. 2019:21:1286-99. https://doi.org/10.1038/s41556-019-0392-4.

24. Thanasopoulou A, Tzankov A, Schwaller J. Potent co-operation between the NUP98-NSD1 fusion and the FLT3-ITD mutation in acute myeloid leukemia induction. Haematologica. 2014;99:1465-71 https://pubmed. ncbi.nlm.nih.gov/24951466. Ferrata Storti Foundation;

25. Kishtagari A, Ng KP, Jarman C, Tiwari AD, Phillips JG, Schuerger C, et al. A first-in-class inhibitor of ISWI-mediated (ATP-dependent) transcription repression releases terminal-differentiation in AML cells while sparing Normal hematopoiesis. Blood. 2018;132:216 LP-216 http://www.blood journal.org/content/132/Suppl_1/216.abstract.

26. Rodrigues CP, Shvedunova M, Akhtar A. Epigenetic regulators as the gatekeepers of hematopoiesis. Trends Genet. 2021;37:125-42. https://doi. org/10.1016/j.tig.2020.09.015 Elsevier.

27. Ostapcuk V, Mohn F, Carl SH, Basters A, Hess D, lesmantavicius V, et al. Activity-dependent neuroprotective protein recruits HP1 and CHD4 to control lineage-specifying genes; 2018.

28. Feric M, Misteli T. Phase separation in genome organization across evolution. Trends Cell Biol. 2021;31:671-85 https://www.sciencedirect.com/ science/article/pii/S0962892421000477.

29. Barisic D, Stadler MB, lurlaro M, Schübeler D. Mammalian ISWI and SWI/ SNF selectively mediate binding of distinct transcription factors. Nature. 2019;569:136-40.

30. Kokavec J, Zikmund T, Savvulidi F, Kulvait V, Edelmann W, Skoultchi Al, et al. The ISWI ATPase Smarca5 (Snf2h) is required for proliferation and differentiation of hematopoietic stem and progenitor cells. Stem Cells. 2017;35:1614-23.

31. Sears RM, May DG, Roux KJ. BiolD as a tool for protein-proximity labeling in living cells. Methods Mol Biol. 2019;2012:299-313 https://pubmed. ncbi.nlm.nih.gov/31161514.

32. Liu X, Salokas K, Weldatsadik RG, Gawriyski L, Varjosalo M. Combined proximity labeling and affinity purification - mass spectrometry workflow for mapping and visualizing protein interaction networks. Nat Protoc. 2020;15:3182-211. https://doi.org/10.1038/s41596-020-0365-x.

33. Shima Y, Yumoto M, Katsumoto T, Kitabayashi I. MLL is essential for NUP98-HOXA9-induced leukemia. Leukemia. 2017;31:2200-10. https:// doi.org/10.1038/leu.2017.62.

\section{Publisher's Note}

Springer Nature remains neutral with regard to jurisdictional claims in published maps and institutional affiliations.

Ready to submit your research? Choose BMC and benefit from:

- fast, convenient online submission

- thorough peer review by experienced researchers in your field

- rapid publication on acceptance

- support for research data, including large and complex data types

- gold Open Access which fosters wider collaboration and increased citations

- maximum visibility for your research: over $100 \mathrm{M}$ website views per year

At BMC, research is always in progress.

Learn more biomedcentral.com/submissions 